\title{
Image Segmentation Using Morphological Watershed Applied to Cartography
}

\author{
Nilcilene das Graças Medeiros, Erivaldo Antonio da Silva, \\ Danilo Aparecido Rodrigues, and José Roberto Nogueira \\ Unesp, Faculdade de Ciências e Tecnologia - Rua Roberto Simonsen, 305 - Presidente \\ Prudente - SP, CEP 19060 - 900 - Brasil \\ \{medeiros, erivaldo\}@prudente.unesp.br
}

\begin{abstract}
Image segmentation is a process frequently used in several different areas including Cartography. Feature extraction is a very troublesome task, and successful results require more complex techniques and good quality data. The aims of this paper is to study Digital Image Processing techniques, with emphasis in Mathematical Morphology, to use Remote Sensing imagery, making image segmentation, using morphological operators, mainly the multi-scale morphological gradient operator. In the segmentation process, pre-processing operators of Mathematical Morphology were used, and the multi-scales gradient was implemented to create one of the images used as marker image. Orbital image of the Landsat satellite, sensor TM was used. The MATLAB software was used in the implementation of the routines. With the accomplishment of tests, the performance of the implemented operators was verified and carried through the analysis of the results. The extration of linear feature, using mathematical morphology techniques, can contribute in cartographic applications, as cartographic products updating. The comparison to the best result obtained was performed by means of the morphology with conventional techniques of features extraction.
\end{abstract}

\section{Introduction}

Effective procedures on images segmentation are required for the successful achievement of the features extraction stage, which are of great interest of several areas, but in the specific case of the cartography area, for features identification and updating.

This work assembles the use of Remote Sensing data and Image Digital Processing techniques, especially the Mathematical Morphological toolbox in the features semiautomatic extration.

The features extraction process is, in this case, established with the use of morphological operators for the images segmentation. The main morphological segmentation tool is based on the transformation that defines the waters divisor line (WDL) or watershed of a function [1][2].

The use of the watershed operator in orbital images generates results with oversegmentation, mainly coming from textures existing in such images.

In order to attenuate the excess of segments, a technique used on the generation of marker images, which is one of the input parameters in the used watershed function, was studied, implemented and used in this work. The technique used was the multiscale morphological gradient. 


\section{Mathematical Morphology}

According to [3], the mathematical morphology includes the area that deals with structural and topological properties of objects from their images. This way, the several techniques employed in this area, especially when the image continuous domain is concerned, are similar to some of the techniques employed for geometric modeling.

This study is performed from the structuring element, which is characterized as a completely known and defined set (shape, size). Such set is compared, from a transformation, to the image unknown set.

The mathematical morphology is composed of two basic operators: erosion and dilatation. Both operators, once applied separately, are transformations not always enhancing the images characteristics. Anyway, they allow us to construct some very interesting functions, as an example, the morphological gradient, the opening, the closing, the segmentation and others.

\subsection{Morphological Segmentation}

The current methods for image segmentation basically use two ideas: One of them is finding the contour of objects in the image. The other is assembling points with similar characteristics so that the object of interest is fully reconstructed. The problem of contour identification may be solved with the use of the watershed operator, which is the main morphological segmentation tool, also known as water line.

An intuitive idea of the watershed notion may be composed considering the gray levels image as a topographic surface and assuming that holes have been perforated in each regional minimum of the surface. The surface is then slowly immerged into water. Starting from the minimum at the lowest altitude, the water will progressively flood the retention basins of the image. In addition, dams are raised at the places where the waters coming from two different minimums would emerge. At the end of this flooding procedure, each minimum is surrounded by dams delineating its associated retention basin. The whole set of dams correspond to the watersheds. They provide us with a partition of the input image into its different retention basins [2].

According to [4], watershed associated to the regional minimums set $\mathbf{M}=\bigcup_{i \in \Re} m_{i}$ of an image $S$ may be defined as the union complement of all retention basins $\mathrm{C}_{f}\left(\mathrm{~m}_{\mathrm{i}}\right)$, and it is expressed through the following equation:

$$
\mathrm{WL}(\mathrm{f})=\left[\bigcup_{i \in \mathfrak{R}} C_{f}\left(m_{i}\right)\right]^{c}
$$

where: $m_{\mathrm{i}}$ : regional minimum and $C_{f}\left(m_{i}\right)$ : retention basin.

\subsection{Morphological Multi-scale Gradient}

According to [2], if the size of the structuring element is greater than 1 (mask $3 \times 3$ ), the morphological gradients are defined as thick gradients, which may be expressed through the equation 2 :

$$
\rho_{n B}=\delta_{n B}-\varepsilon_{n B}
$$

where: $\delta_{\mathrm{nB}}$ represents the dilation operator, with structuring element of size $n$; $\varepsilon_{\mathrm{nB}}$ represents the erosion operator, with structuring element of size $n$. 
The thick gradients are recommended when transitions between objects are smooth. However, such gradients establish thick edges, this way loosing the original location of such edges.

Besides, when the distance between two boudaries of a region is shorter than the width of the structuring element, the resulting edges become one. Both problems can be avoided with the use of the morphological multi-scale gradient.

The thickess of the edges obtained from a thick gradient of size $n$ can be reduced by an erosion $\mathcal{E}$ with element of size "n-1".

According to [2], when thick gradients are originated from two different united edges, the resulting thickness is greater than the concerned structuring element width. Those regions may be removed through the use of the white top-hat (WTH) operator by size $n$, which is then followed by an erosion of size $n-1$ to output a thin edge at scale $n$.

The magnitude of the resulting edges is smaller than the magnitude corresponding to the edge's size morphological gradient. The solution is found by adjusting pixels different from zero from the eroded white top-hat for their original value in the thick gradient image. This way, the morphological gradient is defined for the scale $n$, which is by $\rho^{*}{ }_{n B}$ [2], given by the expression 3 :

$$
\rho_{n B}^{*}=\rho_{n B} * \mathrm{~T}_{\left[1, t_{\max }\right]} \varepsilon_{(n-1) B} W T H_{n B} \rho_{n B}
$$

where $\rho_{n B}$ is conventional morphologic gradient and $\mathrm{T}_{[1, \operatorname{tmax}]}$ is the threshold.

The edges map for all scales is obtained by calculating the maximum between $\rho_{n B}^{*}$ for all $n$, which is expressed through the equation 4 .

$$
\rho^{*}=\underset{n B}{\vee} \rho_{n B}^{*}
$$

where $\underset{n B}{\vee}$ represents the maximum operation for all $n$.

So we call $\rho^{*}$ the non-parametric multi-scale morphological gradient. Figure 1 illustrates an example of a multi-scale gradient.
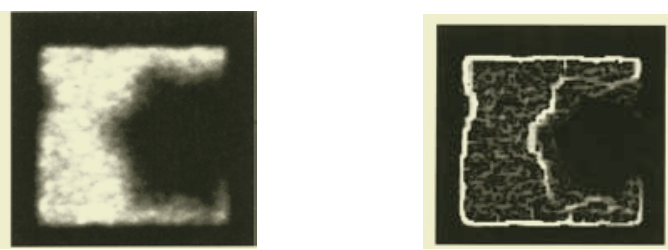

Fig. 1. Orbital image (a), multi-scale morphological gradient obtained through the maximum calculation of all multi-scale morphological gradiente $\rho^{*}{ }_{n B}(\mathrm{~b})$

The multi-scale gradient allows us to enhance edges of each scale, comparing edges only belonging to a certain scale; in other words, such tool evidences the edges belonging to the original image. This way, the information of interest is better evidenced. 


\section{Methodology}

For the development and achievemet of routines concerning the watershed segmentation, was used the image corresponding to the track of the international airport Antonio Carlos Jobim (Galeão), on 8/1/1987 (path 271, Row 76) from Landsat/TM satellite.

The treatment of the input image in the watershed function was performed in this work through morphological filters. In order to generate the markers images, the achievement of the multi-scale morphological gradient technique, in the MATLAB software environment was performed.

Besides the dilation and erosion operators, white top-hat and threshold were used for the development of the multi-scale gradient, also achieved. Such operator's implemented function requires four parameters, which are the input image, the number of scales, the threshold value and the struturing element.

The perfomed processing sequence began with the input image filtering through the morphological operators, progressively employing the implemented operator for the marker image generation, and the watershed segmentation function was applied.

\section{Results}

The used watershed segmentation operator has as input parameters, the raw or treated original images, the markers image and the structuring element.

The inlet image used in tests is shown in Figure 2. Figure 3a presents the result of the marker image generated through the multi-scale gradient, processed with 10 scales, threshold 10 and isotropic structuring element. Now figure $3 \mathrm{~b}$ shows the marker image generated through simple morphologic gradient.

Figures $4 \mathrm{a}$ and $4 \mathrm{~b}$ respectively shows the result from the watershed morphological segmentation with multi-scale gradient marker image and the result from the watershed morphological segmentation with the simple morphologic gradient as marker image.

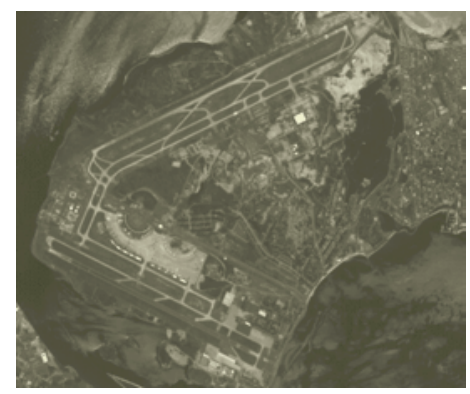

Fig. 2. Original image

The images presented in $3 \mathrm{a}$ and $3 \mathrm{~b}$ were used as marker images and the results obtained are presented in figures $4 \mathrm{a}$ and $4 \mathrm{~b}$.

Figure 4a presents less segmentation when compared to the result obtained in $4 \mathrm{~b}$. When the result obtained in $4 \mathrm{a}$ is analyzed, it is clearly observed that the features of interest are preserved in $4 \mathrm{a}$; the same does not occur in $4 \mathrm{~b}$. 


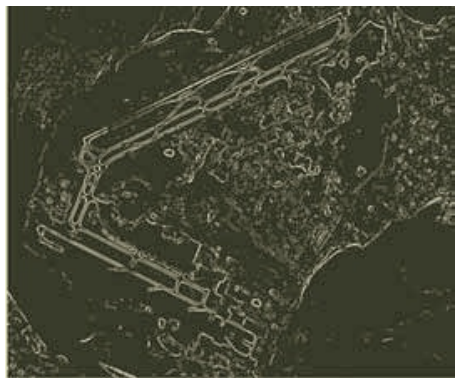

(a)

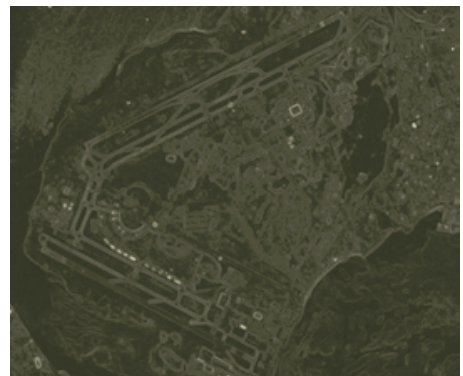

(b)

Fig. 3. Image resulting from the multi-scale gradient (a), image resulting from simple morphological gradient technique (b)

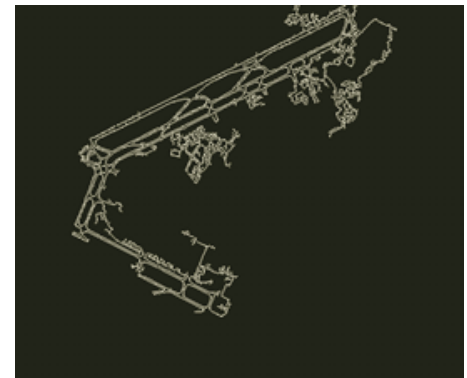

(a)

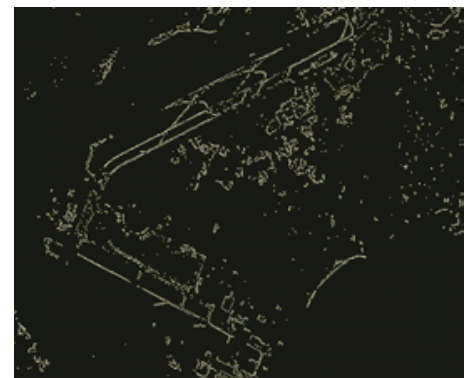

(b)

Fig. 4. Image resulting from the watershed operator with multi-scale gradient (a), image resulting from the watershed operator with simple morphological gradient (b)

In another test, the markers image generated after the original image passed through a pre-processing stage was used. In this stage, morphological operators with the function of removing contrast peaks higher than the stipulated, removing areas smaller than the stipulated and reconstructing the image with infinite interactions of the contrasted image dilation operator in relation to the inlet image were used. Such result is presented in figure 5.

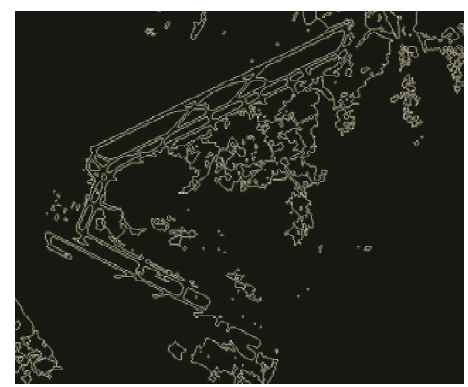

Fig. 5. Image resulting from the watershed operator with pre-process image original as marker

Comparing the results obtained in figures $4 \mathrm{a}$ and 5, one concludes that the result obtained in $4 \mathrm{a}$ remains to be the one presenting the best result of all morphological 
processing used. Such comparison is corroborated when we observe the large amount of segmentation presented in figure 5 .

With regard to the application of morphological operators, the work could be considered as concluded, presenting as the best result, as already explained, the result obtained in figure $4 \mathrm{a}$.

In order for this work to emphasize even more the positive contribution of the use of morphological operators aiming at the extraction of linear features of interest from digital images, a comparison between the best result obtained by means of the morphology to conventional techniques of features extraction was performed.

For this, the Sobel operator was applied to the original image with the purpose of obtaining the markers image in order for the conventional Watershed operator to be applied. The results obtained in this processing are presented in figure 6 .

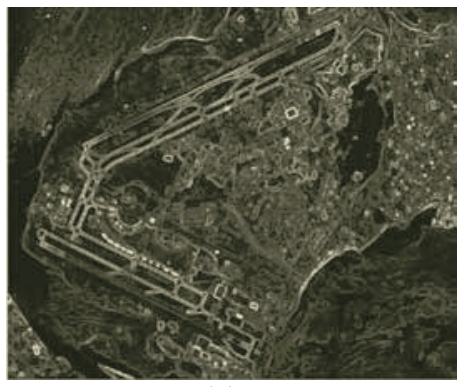

(a)

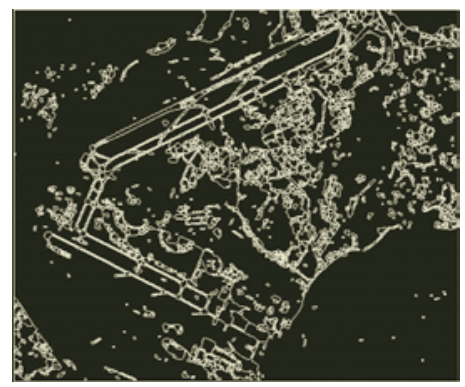

(b)

Fig. 6. Image resulting from the Sobel detector (a), image resulting from the conventional watershed detector with Sobel detector as marker (b)

The Sobel operator was applied with $3 \times 3$ size mask and the conventional watershed operator that best result presented was with initial value of 50 .

As final analysis, the comparison between the best result obtained by means of the morphology (figure $4 \mathrm{a}$ ) to the result obtained through conventional technique (figure 6b) was then performed. From this analysis, it is concluded that the use of the morphology operators presented better result if compared to conventional technique, what corroborates the potential use of the Mathematical morphology in processes of features extraction from digital images.

It is worth emphasizing that in the segmentation process, the treatment performed with the morphological pre-processing operators has fairly improved the final results for the use of the inlet image in the watershed operator, reinforcing the importance of such treatment for the images visual improvement.

\section{Conclusions}

As conclusion for the tests performed, the result obtained with the application of the morphological watershed using as markers image, the result obtained by means of the multi-scale gradient technique was the best in all analyses and comparisons conducted.

Thus, the use of morphological operators showed to be effective for the task of extracting features from digital images. 
It is important emphasizing that the improvement on the results obtained was achieved partly by the treatment performed in the raw image from the pre-processing for the inlet image in the watershed operator process, improving such information may bring on significant results.

The objective of this paper, which was to search for better results with the multiscale gradient approach, was achieved, bringing positive contributions for the segmentation processes, where watershed is employed.

From all tests performed, even results not presented, but still supporting the analysis, we conclude that the results of applications with the implemented morphological operators were better.

Thus, the morphological segmentation will be able to be employed as an semiautomatic alternative method in features extractions for targets identification tasks or in the cartographic products updating.

\section{Acknowledgements}

This work was subsidized by the CNPq and FAPESP, both Brazilian Foundations to Research.

\section{References}

1. Meyer, F., Beucher, S.: The morphological approach to segmentation: the watershed transformation. In Dougherty, E. R. Mathematical Morphology in Image Processing. Marcel Dekker, New York. (1993) 433-481

2. Soille, P.: Morphological Image Analysis - Principles and Applications. In Springer-Verlag Berlin Heidelberg (1999) 316

3. Gomes, J., Velho, L.: Computação Gráfica: Imagem. Série de Computação e Matemática, SBM/IMPA (1995)

4. Préteux, F.: On a distance function approach for grey-level mathematical morphology. In Dougherty E.R. Mathematical Morphology in Image Processing. Marcel Dekker, New York (1993) 323-351 